\title{
DEFINITION OF THE ABELIAN, THE TWO HYPOABELIAN, AND RELATED LINEAR GROUPS AS QUOTIENT-GROUPS OF THE GROUPS OF ISOMORPHISMS OF CERTAIN ELEMENTARY GROUPS*
}

$3 \mathrm{Y}^{2}$

\section{LEONARD EUGENE DICKSON}

1. The present paper aims to give a natural definition of the Abelian and two hypoabelian groups, which moreover preserves the essence of JORDAN's definition based upon his important, but artificial, conception of "exposants d'échange" (Traité, pp. 179, 195). A second formal definition, by means of the invariants (11), (14) and (21) below, may be obtained from Jordan (l. c. p. 217 and pp. $438-440)$.

Following in the main the developments of Jordan (l. c. pp. 420-447, in particular) on the construction of solvable groups, we may obtain the above groups as quotient-groups of the groups of isomorphisms of certain elementary groups. $\dagger$ In May, 1898, I communicated such a treatment to Professor Moore, who emphasized the desirability of presenting the definitions thus obtained for the Abelian and hypoabelian groups in two distinct ways: viz., from the standpoint $\ddagger$ of JoRDAN's linear groups and from the standpoint $\$$ of abstract groups.

* Presented to the Society at the Columbus meeting Aug. 26, 1899 . Received for publication Oct. 1, 1899.

For cross-reference and abstract, cf. Bulletin, April, 1899, pp. 331-2 (where p. 331, line 2 from bottom, for holoedrically one should read meriedrically).

$\dagger$ For an outline, see $\xi \xi 8-10$ below. A different aim being in view, the details of the calculation become much simpler than those of JoRDAN.

$\ddagger$ For example, JoRDAN (1. c. $\xi \xi$ 118-119) obtains the non-homogeneous linear group as the largest group of literal substitutions transforming into itself the commutative literal group readily defined by means of the linear substitutions modulo $p$,

$$
x_{i}^{\prime} \equiv x_{i}+a_{i} \quad(i=1, \cdots, n) .
$$

\& The idea of the group of isomorphisms of an abstract group seems to have been arrived at independently by MOORE and HöLDER, the former applying it to define the linear homogeneous group as the group of transformation into itself of the Abelian group of type $\{1,1, \cdots, 1\}_{n}$. See his article in the Bulletin of the American Mathematical Society, Nov., 1895.

I must however refer to the paper by EnRICo BETT, Sopra la teorica delle sostituzioni, A n nali di Scienze Matematiche e Fisiche, pp. 5-34, 1855 . He obtains a group equivalent to the homogeneous linear group as the "massimo Moltiplicatore" of a cyclic group of order 
Quite recently I have made the investigation from the latter point of view and have found the method so much simpler and more desirable that $I$ have abandoned my earlier work, which was not fully complete, and give in $\S \S 8-10$ a mere outline of it.

2. Consider the group $F$ generated by the operators of period $p$ ( $p$ being prime),

with the generational relations,*

$$
\Theta, A_{i}, B_{i}
$$

$$
(i=1, \cdots, m)
$$

$$
\begin{aligned}
& \Theta^{p}=1, A_{i}^{p}=1, B_{i}^{p}=1 \\
& (i=1, \cdots, m) \\
& \left\{\begin{array}{llrl}
\Theta A_{i}=A_{i} \Theta, & \Theta B_{i}=B_{i} \Theta & (i=1, \cdots, m) \\
A_{i} A_{j}=A_{j} A_{i}, & B_{i} B_{j}=B_{j} B_{i} & (i, j=1, \cdots, m) \\
A_{i} B_{j}=B_{j} A_{i}, & A_{i} B_{i}=\Theta B_{i} A_{i} & \left(\begin{array}{c}
i, j=1, \cdots, m \\
i \neq j
\end{array}\right) .
\end{array}\right.
\end{aligned}
$$

It follows at once that

$$
A_{i}^{x} B_{i}^{y}=\Theta^{x y} B_{i}^{y} A_{i}^{x}
$$

and hence, in general, if we set

$$
\begin{gathered}
S \equiv \Theta^{t} A_{1}^{x_{1}} B_{1}^{y_{1}} \cdots A_{m}^{x_{m}} B_{m}^{y_{m}}, \\
\Sigma \equiv \Theta \tau A_{1}^{\xi_{1}} B_{1}^{\eta_{1}} \cdots A_{m}^{\xi_{m}} B_{m}^{\eta_{m}}, \\
S \Sigma=\Theta^{\left.\sum_{i=1}^{m} x_{i} \eta_{i}-\xi_{i} y_{i}\right)} \Sigma S,
\end{gathered}
$$

a relation which includes all of the relations (2).

By application of (2) any operator of $F$ may be put into the form $S$ above. Since $A_{i}$ is not expressible in terms of the remaining generators, and likewise for $B_{i}$, it follows that $S=1$ if and only if

$$
x_{i} \equiv y_{i} \equiv t \equiv 0(\bmod p)
$$$$
(i=1, \cdots, m)
$$

Further, $S=\Sigma$ requires that

$$
x_{i} \equiv \xi_{i}, \quad y_{i} \equiv \gamma_{i}, \quad t \equiv \tau(\bmod p) .
$$

Indeed, $S \Sigma^{-1}=1$ may be given the form

$$
\Theta^{t-\tau+\sum_{i=1}^{m} \xi_{i}\left(y_{i}-\eta_{i}\right)} A_{1}^{x_{1}-\xi_{1}} B_{1}^{y_{1}-\eta_{1}} \cdots A_{m}^{x_{m}-\xi_{m}} B_{m}^{y_{m}-\eta_{m}}=1 .
$$

$p^{\nu}$. BETTI observes (p. 34) that this result was given by GaLoIs without proof. Although I have not found the passage in the papers of GALOIS referred to by BETTI, nevertheless I am confident that GaLOIs had the conception of the group of isomorphisms of the Abelian group of type $\{1,1, \cdots 1\}_{n}$, at least for $n=2$. See the passage at the bottom of p. 58 of the fragment preserved to us of the posthumous paper, Des équations primitives qui sont soluble par radicaux, Oeuvres Mathématiques $D^{\prime}$ Évariste Galois, Paris, 1897.

* The relation $\theta^{p}=1$ follows from (3); thus $A_{i} B_{i}^{p}=\theta^{p} B_{i}^{p} A_{i}$. We may evidently drop $\theta$ from the list of generators. 
It follows from (4) that the powers of $\Theta$ are the only operators of $F$ which are commutative with every operator of $F$.

We may prove by induction the formula

$$
S^{k}=\Theta^{k t-1 / 2 k(k-1) \sum_{i=1}^{m} x_{i} y_{i}} A_{1}^{k x_{1}} B_{1}^{k y_{1}} \cdots A_{m}^{k x_{m}} B_{m}^{k y_{m}}
$$

Hence, for $p>2$, every operator of $F$ has the period $p$ and, for $p=2$, the period 2 or 4 , viz.,

$$
S^{2}=\Theta^{\Sigma x_{i} y_{i}}
$$

3. Every isomorphism $I$ of the group $F$ into itself is obtained by introducing a new set of generators

$$
\Theta^{\prime}, A_{i}^{\prime}, B_{i}^{\prime}
$$$$
(i=1, \cdots, m)
$$

satisfying the relations (1) and (2) and capable of generating the entire group $F$. We may set

$$
\left\{\begin{array}{l}
\Theta^{\prime}=\Theta^{s} \\
A_{i}^{\prime}=\Theta^{l_{i}} A_{1}^{a_{1 i}} B_{1}^{b_{1 i}} \cdots A_{m}^{a_{m i}} B_{m}^{b_{m i}} \\
B_{i}^{\prime}=\Theta^{r_{i}} A_{1}^{c_{1 i}} B_{1}^{d_{1 i}} \cdots A_{m}^{c_{m i}} B_{m}^{d_{m i}}
\end{array}\right.
$$

where $s \neq 0(\bmod p)$.

Since $\Theta^{\prime}, A_{i}^{\prime}, B_{i}^{\prime}$ shall generate $F$, we must be able, for arbitrarily given values of $t^{\prime}, x_{i}^{\prime}, y_{i}^{\prime}$, to determine $t, x_{i}, y_{i}$ so that

$$
\Theta^{i} A_{1}^{x_{1}^{\prime}} B_{i}^{y_{1}^{\prime}} \cdots \equiv \Theta^{\prime t} A_{1}^{\prime x_{1}} B_{1}^{\prime y_{1}} \cdots
$$

Replacing $\Theta^{\prime}, A_{i}^{\prime}, B_{i}^{\prime}$ by their values (6) and applying (2) to bring to the left of the product the operators $A_{1}$, and next the $B_{1}$, etc., we obtain the condition

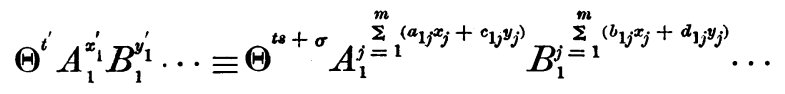

where the exact expression for $\sigma$ is immaterial. It follows from $\S^{2}$ that the corresponding exponents must be congruent modulo $p$. Thus $t$ is determined uniquely from

$$
t^{\prime} \equiv t s+\sigma(\bmod p) .
$$

We have further the following $2 m$ conditions

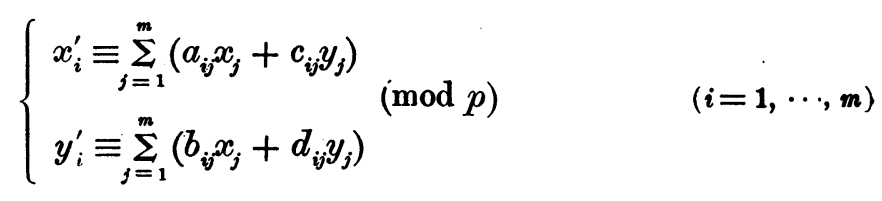


For $x_{i}^{\prime}, y_{i}^{\prime}$ arbitrary, the congruences (9) can be solved for $x_{i}, y_{i}$ (and then uniquely) if and only if the determinant

$$
D \equiv\left|\begin{array}{cccc}
a_{11} & c_{11} & \cdot & \cdot \\
b_{11} & d_{11} & . & \cdot \\
\cdot & \cdot & . & .
\end{array}\right| \neq 0(\bmod p) .
$$

We next seek the conditions under which the new generators (6) satisfy the generational relations (2) or the equivalent single relation (4). $S$ denoting the operator (7) and $\Sigma$ the analogous operator

$$
\Sigma \equiv \Theta^{\tau^{\prime}} A_{1}^{\xi_{1}^{\prime}} B_{1}^{\eta_{1}^{\prime}} \cdots \equiv \Theta^{\prime \tau} A_{1}^{\prime \xi_{1}} B_{1}^{\prime \eta_{1}} \cdots
$$

the relation (4) requires

$$
\Theta^{\sum_{i=1}^{m}\left(x_{i}^{\prime} \eta_{i}^{\prime}-\xi_{i}^{\prime} y_{i}^{\prime}\right)}=\Theta^{\sum_{i=1}^{m}\left(x_{i} \eta_{i}-\xi_{i} y_{i}\right)}
$$

Since $\Theta^{\prime}=\Theta^{s}$, the substitution (9) and the cogredient substitution on the $\xi_{i}, \eta_{i}$ must have the simultaneous relative invariant

$$
\sum_{i=1}^{m}\left(x_{i} \eta_{i}-\xi_{i} y_{i}\right)
$$

that is, must satisfy the relation

$$
\sum_{i=1}^{m}\left(x_{i}^{\prime} \eta_{i}^{\prime}-\xi_{i}^{\prime} y_{i}^{\prime}\right) \equiv s \sum_{i=1}^{m}\left(x_{i} \eta_{i}-\xi_{i} y_{i}\right)
$$

This relation imposes upon the coefficients the following relations modulo $p$ :

$$
\left\{\begin{array}{ll}
\sum_{i=1}^{m}\left|\begin{array}{ll}
a_{i j} & c_{i j} \\
b_{i j} & d_{i j}
\end{array}\right| \equiv s, & \sum_{i=1}^{m}\left|\begin{array}{cc}
a_{i j} & c_{i k} \\
b_{i j} & d_{i k}
\end{array}\right| \equiv 0, \\
\sum_{i=1}^{m}\left|\begin{array}{ll}
a_{i j} & a_{i k} \\
b_{i j} & b_{i k}
\end{array}\right| \equiv 0, & \sum_{i=1}^{m}\left|\begin{array}{ll}
c_{i j} & c_{i k} \\
d_{i j} & d_{i k}
\end{array}\right| \equiv 0,
\end{array} \quad(j, k=1, \cdots, m ; j \neq k) .\right.
$$

From the manner of our derivation of (12) or by direct verification, we observe that (12) form the sufficient (as well as necessary) conditions in order that $\Theta^{\prime}, A_{i}^{\prime}, B_{i}^{\prime}$ shall satisfy the relations (2).

It remains to require that $A_{i}^{\prime}$ and $B_{i}^{\prime}$ have the period $p$. By the theorem at the end of $\S 2$, no new condition is imposed if $p>2$; while for $p=2$, we have the conditions

$$
\sum_{j=1}^{m} a_{j i} b_{j i} \equiv \sum_{j=1}^{m} c_{j i} d_{j i} \equiv 0(\bmod 2) \quad(i=1, \cdots, m) .
$$

By the same theorem, we find by squaring (7) that the substitution (9) must, for $p=2$, satisfy the relation

$$
\sum_{i=1}^{m} x_{i}^{\prime} y_{i}^{\prime} \equiv s \cdot \sum_{i=1}^{m} x_{i} y_{i}(\bmod 2) \text {. }
$$

Trans. Am. Math. Soc., 3. 
Since $s=1$ for $p=2$, we find that (14) imposes upon the coefficients of (9) precisely the conditions (12) and (13).

We have now proved the following:

Theorem.-The set of generators (6) will define an isomorphism I of the group $F$ into itself if, and only if, the exponents satisfy the relations (10), (12) and, for $p=2$, also (13). To every isomorphism I corresponds a $2 m$ ary linear homogeneous substitution (9) belonging to the general Abelian group if $p>2$ and to the first hypoabelian group if $p=2$; and inversely, every such substitution (9) is so obtainable.

4. No condition is imposed upon the exponents $l_{i}, r_{i}$ in order that (6) define an isomorphism $I$. Moreover, it is evident that the set of generators

$$
\Theta^{\prime}=\Theta, \quad A_{i}^{\prime}=\Theta^{\prime i} A_{i}, \quad B_{i}^{\prime}=\Theta^{r_{i}} B^{i} \quad(i=1, \cdots, m)
$$

defines an isomorphism $I_{1}$ for which the corresponding substitution (9) is the identity. Inversely, when (9) is the identity, the isomorphism is one of the $p^{2 m}$ isomorphisms $I_{1}$. Since the group of the $I$ 's is isomorphic to the group of the corresponding substitutions (9), the group of the $I_{1}$ 's is self-conjugate under the group of the $I$ 's. We have thus the following:

ThEOREM.-The group of isomorphism $I$ of the group $F$ has an invariant sub-group of order $p^{2 m}$ formed by the isomorphisms $\left(6_{1}\right)$. The quotient-group is simply isomorphic to the general Abelian group, if $p>2$, and to the first hypoabelian group, if $p=2$, each on $2 m$ indices taken modulo $p$.

5. Consider the group $F_{1}$ obtained by extending the group $F$ by an operator $J$ commutative with every operator of $F$ and such that $J^{p}=\Theta . \quad J$ is thus of period $p^{2}$. Every operator of $F_{1}$ may be given the form

$$
S \equiv J^{t} A_{1}^{x_{1}} B_{1}^{y_{1}} \cdots A_{m}^{x_{m}} B_{m}^{y_{m}},
$$

which reduces to the identity if and only if

On setting

$$
x_{i} \equiv y_{i} \equiv 0(\bmod p), \quad t \equiv 0\left(\bmod p^{2}\right) .
$$

$$
\Sigma \equiv J^{\tau} A_{1}^{\xi_{1}} B_{1}^{\eta_{1}} \cdots A_{m}^{\xi_{m}} B_{m}^{\eta_{m}},
$$

the relation (4) holds true. Further, $S=\Sigma$ requires that

$$
x_{1} \equiv \xi_{i}, \quad y_{i} \equiv \eta_{i}(\bmod p), \quad t \equiv \tau\left(\bmod p^{2}\right) .
$$

We readily verify the formula

$$
S^{k}=J^{k t} \Theta^{-y_{2} k(k-1) \Sigma x_{i} y_{i}} A_{1}^{k x_{1}} B_{1}^{k y_{1}} \cdots A_{m}^{k x_{m}} B_{m}^{k y_{m}} .
$$

Hence, according as $p>2$ or $p=2$, we have

$$
S^{p}=\Theta^{t} \text { or } S^{p}=\Theta^{t-\Sigma x_{i} y_{i}} .
$$


By (4) the powers of $J$ are the only operators of $F_{1}$ commutative with every operator of $F_{1}$. Every isomorphism of $F_{1}$ into itself is therefore obtained by introducing new generators of the form

$$
\left\{\begin{array}{l}
J^{\prime}=J^{s} \\
A_{i}^{\prime}=J^{l_{i}} A_{1}^{a_{1 i}} B_{1}^{b_{1 i}} \cdots A_{m}^{a_{m i}} B_{m}^{b_{m i}} \\
B_{i}^{\prime}=J^{r_{i}} A_{1}^{c_{1 i}} B_{1}^{d_{1 i}} \cdots A_{m}^{c_{m i}} B_{m}^{d_{m i}} .
\end{array}\right.
$$

As in $\S 3, J^{\prime}, A_{i}^{\prime}, B_{i}^{\prime}$ will generate the whole group $F_{1}$ if, and only if, (10) holds and will satisfy the generational relation (4) if, and only if, the conditions (12) be satisfied. Finally, by (16), $A_{i}^{\prime}$ and $B_{i}^{\prime}$ will be of period $p$ if, and only if,

$$
\left\{\begin{array}{lll}
\text { for } p>2, & l_{i} \equiv r_{i} \equiv 0(\bmod p) & (i=1, \cdots, m), \\
\text { for } p=2, & l_{i}-\sum_{j=1}^{m} a_{j i} b_{j i} \equiv r_{i}-\sum_{j=1}^{m} c_{j i} d_{j i} \equiv 0(\bmod 2) & (i=1, \cdots, m) .
\end{array}\right.
$$

For $p>2$, we set

$$
l_{i}=p \lambda_{i}, r_{i}=p \rho_{i} \quad(i=1, \cdots, m) .
$$

The most general suitable set of the new generators is then

$$
\left\{\begin{array}{l}
J^{\prime}=J^{s}, \Theta^{\prime}=\Theta^{s}, \\
A_{i}^{\prime}=\Theta^{\lambda_{i}} A_{1}^{a_{1 i}} B_{1}^{b_{1 i}} \cdots, B_{i}^{\prime}=\Theta^{\rho_{i}} A_{1}^{c_{1 i}} B_{1}^{d_{1 i}} \cdots
\end{array}\right.
$$

where $a_{j i}, b_{j i}, c_{j i}, d_{j i}$ satisfy $(10),(12)$, while $\lambda_{i}$ and $\rho_{i}$ are arbitrary integers.

For $p=2$, we set

$$
l_{i}=2 \lambda_{i}+\sum_{j=1}^{m} a_{j i} b_{j i}, \quad r_{i}=2 o_{i}+\sum_{j=1}^{m} c_{j i} d_{j i} .
$$

The most general set of new generators is then

$$
\left\{\begin{array}{l}
J^{\prime}=J^{s}, \Theta^{\prime}=\Theta^{s} \\
A^{i}=\Theta^{\lambda_{i}} J^{\Sigma a_{j i} b_{j i}} A_{1}^{a_{1 i}} B_{1}^{b_{1 i}} \cdots \\
B_{i}^{\prime}=\Theta^{\rho_{i}} J^{\Sigma c_{j i} d_{j i}} A_{1}^{c_{1 i}} B_{1}^{d_{1 i}} \cdots
\end{array}\right.
$$

For $p \equiv 2$, the new set of generators

$$
J^{\prime}=\Theta^{\tau} J, \Theta^{\prime}=\Theta, A_{i}^{\prime}=\Theta^{\lambda_{i}} A_{i}, B_{i}^{\prime}=\Theta^{\rho_{i}} B_{i} \quad(i=1, \cdots, m)
$$

defines an isomorphism for which the corresponding substitution (9) is the identity. Inverselý, if the substitution be the identity, the set of generators must be of the above kind. We may state the following:

THEOREM.-The group of isomorphisms of the group $F_{1}$ has an invariant subgroup of order $p^{2 m+1}$ formed by the isomorphisms (a). The quotient-group is simply isomorphic with the general Abelian group on $2 m$ indices taken modulo $p$. 
6. Consider the group $F^{\prime}$ generated by

$$
\Theta, A_{i}, B_{i}
$$

$(i=1, \cdots, m)$

whose periods are defined by the relations

$$
A_{1}^{p}=B_{1}^{p}=\Theta, \quad \Theta^{p}=A_{i}^{p}=B_{i}^{p}=1 \quad(i=2,3, \cdots, m),
$$

and whose further generational relations are given by (2). The general operator of $F^{\prime}$ may be given the form

$$
S \equiv \Theta^{t} A_{1}^{x_{1}} B_{1}^{y_{1}} \cdots A_{m}^{y_{m}} B_{m}^{y_{m}}
$$

where each exponent may be supposed to be less than $p$. In this reduced form,* $S$ is the identity if and only if the exponents are all zero.

Two general operators $S$ and $\Sigma$ of the group $F^{\prime}$ satisfy the generational relation (4). Formula (5) evidently holds for the group $F^{\prime}$. Applying (19) to (5) we obtain for the period of an operator $S$ of $F^{\prime}$ the formulæ

$$
\begin{cases}S^{p}=\Theta^{x_{1}+y_{1}} & (\text { if } p>2), \\ S^{p}=\Theta^{x_{1}+y_{1}-\sum_{i=1}^{m} x_{i} y_{i}} & \text { (if } p=2) .\end{cases}
$$

To obtain the group of isomorphisms of $F^{\prime}$, we proceed as in $\S 3$, the only variation being in regard to the periods of $A_{i}^{\prime}, B_{i}^{\prime}$. Raising the identity (7) to the $p^{\text {th }}$ power and applying (20), we obtain the periodicity conditions,

$$
\begin{array}{ll}
\Theta^{x_{1}^{\prime}+y_{1}^{\prime}}=\Theta^{\prime x_{1}+y_{1}} & (\text { if } p>2) . \\
\Theta^{x_{1}^{\prime}+y_{1}^{\prime}-\Sigma x_{i}^{\prime} y_{i}^{\prime}}=\Theta^{\prime x_{1}+y_{1}-\Sigma x_{i} y_{i}} & (\text { if } p=2),
\end{array}
$$

For $p=2, \Theta^{\prime} \equiv \Theta^{s}=\Theta$, so that the condition is

$$
x_{1}^{\prime}+y_{1}^{\prime}+\sum_{i=1}^{m} x_{i}^{\prime} y_{i}^{\prime} \equiv x_{1}+y_{1}+\sum_{i=1}^{m} x_{i} y_{i}(\bmod 2) \text {, }
$$

which imposes upon the coefficients of the substitution (9) the conditions (12) together with the following conditions modulo 2 (where $\delta_{11}=1, \delta_{12}=\cdots=\delta_{1 m}$ $=0), \uparrow$

$$
a_{1 j}+b_{1 j}+\sum_{i=1}^{m} a_{i j} b_{i j} \equiv c_{1 j}+d_{1 j}+\sum_{i=1}^{m} c_{i j} d_{i j} \equiv \delta_{1 j}
$$$$
(j=1, \ldots, m) \text {. }
$$

As one sees directly from (20), the conditions (22) suffice when $p=2$ to give $A_{i}^{\prime}$ and $B_{i}^{\prime}$ the periods (19). For $p=2$, the group of substitutions (9) having the invariant (21) is the second hypoabelian group. Proceeding as in $\S 4$, we have the following result:

* Unless so reduced, $S \equiv 1$ if and only if

$$
x_{i} \equiv y_{i} \equiv t \equiv 0(\bmod p), \quad x_{1}+y_{1}+p t \equiv 0\left(\bmod p^{2}\right)
$$

† The relation (21) is not a formal, but a numerical, identity. Indeed, we must note that $x^{2} \equiv x(\bmod 2)$. 
TheOREM.-The group of isomorphisms of the group $F^{\prime \prime}$ has an invariant subgroup of order $p^{2 m}$ formed by the isomorphisms $\left(6_{1}\right)$. Their quotient-group is simply isomorphic, for $p=2$, to the second hypoabelian group, and, for $p>2$, to that subgroup of the general Abelian group satisfying the relation

$$
x_{1}^{\prime}+y_{1}^{\prime} \equiv s\left(x_{1}+y_{1}\right)
$$

where $s$ is any integer not divisible by $p$, but the same as in (12).

Since the Abelian group contains the substitution $L_{1}$, which alters only $x_{1}$, replacing it by $x_{1}+y_{1}$, the subgroup defined by (23) is conjugate within the general Abelian group to that subgroup which multiplies $x_{1}$ by the parameter $s$.

7. Consider, finally, the group $F_{1}^{\prime}$ obtained by extending $F^{\prime}$ by an operator $J$ commutative with every operator of $F^{\prime}$ and such that $J^{p}=\Theta$. To obtain the group of isomorphisms of $F_{1}^{\prime}$, we proceed as in $\S 5$. The conditions that the new generators (17) shall have the periods (19) are seen, on applying (15), to be as follows :

$$
\begin{array}{cc}
(\text { for } p>2) & l_{i}+a_{1 i}+b_{1 i} \equiv r_{i}+c_{1 i}+d_{1 i} \equiv \delta_{1 i} \\
(\text { for } p=2) & l_{i}+a_{1 i}+b_{1 i}-\sum_{j=1}^{m} a_{j i} b_{j i} \equiv r_{i}+c_{1 i}+d_{1 i}-\sum_{j=1}^{m} c_{j i} d_{j i} \equiv \delta_{1 i} \\
(i=1, \cdots, m) .
\end{array}
$$

The exponents $l_{i}, r_{i}$ are thus determined modulo $p$. Proceeding as in $\S 5$, we reach the following result :

THEOREM.-The group of isomorphisms of $F_{1}^{\prime}$ has an invariant subgroup of order $p^{2 m+1}$, the quotient-group being simply isomorphic with the general Abelian group on $2 m$ indices modulo $p$.

8. We may give a concrete representation * of the operators of the groups $F, F_{1}, F^{\prime}, F_{1}^{\prime}$, as linear homogeneous substitutions on $p^{m}$ variables $Z_{\xi_{1}}, \ldots, \xi_{m}$ the indices $\xi_{i}$ being taken modulo $p$.

For the groups $F$ and $F_{1}$ we may take

$$
\begin{cases}A_{i}: & Z_{\xi_{1}}^{\prime} \ldots \xi_{m}=\theta^{\xi_{i}} Z_{\xi_{1}} \ldots \xi_{m}, \\ B_{i}: & Z_{\xi_{1}}^{\prime} \ldots \xi_{i} \ldots \xi_{m}=Z_{\xi_{1}} \ldots \xi_{i}+\ldots \xi_{m}, \\ \Theta: & Z_{\xi_{1}}^{\prime} \ldots \xi_{m}=\theta Z_{\xi_{1} \cdots \xi_{m}}, \\ J: & Z_{\xi_{1}}^{\prime} \ldots \xi_{m}=j Z_{\xi_{1}} \ldots \xi_{m},\end{cases}
$$

where $\theta$ is a primitive $p^{\text {th }}$ root of unity and $j$ a primitive root (always existing) of the equation

$$
j^{p}=\theta \text { or } j^{p^{2}}=1 \text {. }
$$

It is readily seen that the substitutions (24) satisfy the relations (1) and (2).

* Taken in a simplified form from JondaN's Traité, $₹ \& 561$ and 566. For JordaN's $\pi$, I use $p$, and avoid the use of his other parameter $p$. 
The general substitution $S$ of $F$ takes the form

$$
Z_{\xi_{1}}^{\prime} \cdots \xi_{m}=\theta^{t+\sum_{i=1}^{m} x_{i}\left(\xi_{i}+y_{i}\right)} Z_{\xi_{1}+y_{1}} \cdots \xi_{m}+y_{m} \text {. }
$$

It is at once evident that two substitutions $S$ are identical if and only if their corresponding exponents $x_{i}, y_{i}, t$ are congruent modulo $p$.

9. By a method similar to that of JoRDAN, l. c. $\S \S 568-9$, but simpler as to details, we can readily prove that the characteristic determinant (with the parameter $K$ ) of (25) is equal modulo $p$ to respectively

$$
\begin{aligned}
& \left(1-K^{p}\right)^{p^{m-1}} \\
& \left(K^{2}-\theta^{\Sigma \cdot r_{i} y_{i}}\right)^{2^{m-1}}
\end{aligned}
$$

Since the substitution $S$ and its transformed (7) by a linear substitution must have equal characteristic determinants, we obtain (14) as a condition necessary for the isomorphism when $p=2$. For the group of transformation of $F_{1}$ into itself, the characteristic determinant of

is, for $p=2$,

$$
J^{t} A_{1}^{\prime x_{1}} B_{1}^{\prime y_{1}} \cdots \equiv J^{\sum_{i=1}^{m}\left(l_{1} x_{i}+r_{i} y_{i}\right)+t} \Theta^{\tau} A_{1}^{x_{1}^{\prime}} B_{1}^{y_{1}^{\prime}} \ldots
$$

$$
\left(K^{2}-\theta^{t+\sum_{i=1}^{m}\left(l_{i} x_{i}+r_{i} y_{i}+x_{i}^{\prime} y_{i}^{\prime}\right)}\right)^{2^{m-1}} .
$$

Hence a condition necessary for the isomorphism is the following,

$$
\sum_{i=1}^{m}\left(x_{i}^{\prime} y_{i}^{\prime}+l_{i} x_{i}+r_{i} y_{i}\right) \equiv \sum_{i=1}^{m} x_{i} y_{i}(\bmod 2),
$$

whence follow the relations $(18)_{p=2}$.

But by this method it remains to discuss the following inverse problem. How many isomorphisms of $F$ (or $F_{1}$ ) into itself correspond to each substitution (9) satisfying the above relations? Compare Jordan, l. c. $\S \S 579-580$.

10. For the groups $F^{\prime \prime}$ and $F_{1}^{\prime}$ of $\S \S 6$ and 7 , we may take

$$
\left\{\begin{array}{l}
A_{1}: \quad Z_{\xi_{1} \cdots \xi_{m}}^{\prime}=j \theta^{\xi_{1}} Z_{\xi_{1}} \cdots \xi_{m} \\
B_{1}: \quad Z_{\xi_{1} \xi_{2} \cdots \xi_{m}}^{\prime}=j Z_{\xi_{1}+1 \xi_{2}} \cdots \xi_{m} \\
\Theta, J, A_{i}, B_{i}(i=2, \cdots, m) \text { defined by (24). }
\end{array}\right.
$$

Then the general substitution $\Theta_{1}^{t}{ }_{1} A^{x_{1}} B^{y_{1}} \ldots$ is

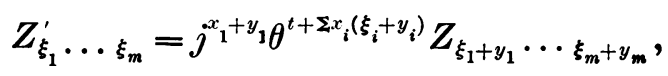

whose characteristic determinant is for $p=2$,

$$
\left(K^{2}-\theta^{x_{1}+y_{1}+\Sigma_{i} y_{i}}\right)^{2^{m-1}} \text {. }
$$

This equals the characteristic determinant of

only if (21) be satisfied.

$$
\Theta^{\prime t} A_{1}^{\prime x_{1}} B_{1}^{\prime y_{1}} \ldots A_{m}^{\prime x_{m}} B_{m}^{\prime y_{m}}
$$

